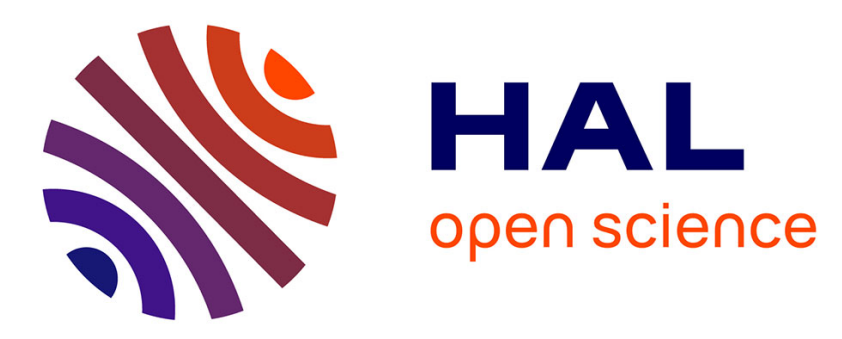

\title{
Trémaux trees and planarity
}

Hubert de Fraysseix, Patrice Ossona de Mendez, Pierre Rosenstiehl

\section{To cite this version:}

Hubert de Fraysseix, Patrice Ossona de Mendez, Pierre Rosenstiehl. Trémaux trees and planarity. International Journal of Foundations of Computer Science, 2006, 17 (5), pp.1017-1029. 10.1142/S0129054106004248 . hal-00097836

\section{HAL Id: hal-00097836 https://hal.science/hal-00097836}

Submitted on 30 Oct 2006

HAL is a multi-disciplinary open access archive for the deposit and dissemination of scientific research documents, whether they are published or not. The documents may come from teaching and research institutions in France or abroad, or from public or private research centers.
L'archive ouverte pluridisciplinaire HAL, est destinée au dépôt et à la diffusion de documents scientifiques de niveau recherche, publiés ou non, émanant des établissements d'enseignement et de recherche français ou étrangers, des laboratoires publics ou privés. 


\title{
Depth-First Search and Planarity
}

\author{
Hubert de Fraysseix, \\ Patrice Ossona de Mendez, \\ Pierre Rosenstiehl \\ Centre d'Analyse et de Mathématiques Sociales (CNRS UMR 8557), \\ École des Hautes Études en Sciences Sociales, 54 boulevard Raspail \\ Paris, 75006, France
}

\begin{abstract}
We present a simplified version of the DFS-based Left-Right planarity testing and embedding algorithm implemented in Pigale [1, 2], which has been considered as the fastest implemented one [3]. We give here a full justification of the algorithm, based on a topological properties of Trémaux trees.
\end{abstract}

\section{Introduction}

It is well known since the publications [4, 5] in 1973-74 by J. Hopcroft and R.E. Tarjan that the time complexity of the problem of graph planarity testing is linear in the number of edges. However, authors of text books and teachers of graph theory know how hard it is to describe and completely justify such algorithms, each one being more tricky than the other. To display a planar embedding is usually a second hard step [6].

A satisfactory graph planarity algorithm should be efficient, clearly justified, and easy to understand. Only a strong mathematical insight of the subject could probably meet these three criteria.

R. Tarjan initiated the use of the Depth-First Search procedure (DFS) to attack planarity testing, both DFS and planarity testing on biconnected graphs being handled recursively. Several authors explained how to extract an embedding during the recursion of such an algorithm. The authors produced in the eighties the so-called LEFT-RIGHT algorithm, a non-recursive version avoiding the 2-connectivity assumption. The LEFT-RIGHT algorithm appeared to be extremely efficient for testing planarity and embedding planar graphs and has been recognized as the fastest among the implemented ones by the comparative tests performed by graph drawing specialists [3]. But even if a tentative mathematical explanation appeared in our papers on Trémaux characterization of planarity [4, 8, 9], it was not enough to fully meet the second and third criteria. 
The code appears in the GPL-licenced software PIGALE [1, 2]. A new, simplified, and faster version has been implemented at the occasion of this paper, which is the one we will discuss here.

In Section 2, Trémaux trees are studied as a mathematical object per se. A rooted tree that spans a graph is a Trémaux tree if each cotree edge is incident to two comparable vertices (with respect to the tree order). It is why cotree edges are then called back-edges. For a given Trémaux tree, the following structural concepts are defined: the low of a vertex or of an edge, the low set of a vertex or of an edge and the fringe of an edge.

Section 3 is devoted to the study of planarity. Planarity has been related to Trémaux trees in [7] in a characterization based on the existence of a bicoloration constrained by three special simple configurations. These configurations are here unified into a single one. In this setting, it is proved that some additional constraints may be imposed, which do not change the existence of a bipartition but lead a simple planar embedding.

The planarity testing and embedding algorithm is then described in Section 4 . It is shown that linear time-complexity is reached by implicitly building a spanning arborescence in the graph of constraints. It shall be noticed that the data structures used by the algorithm are almost trivial ones, which may explain its computational efficiency and the ease to follow the algorithm step by step on an example.

In the sequel, by a "graph" we mean a "connected, loopless multigraph", unless we state it otherwise.

\section{Trémaux Trees}

Depth-first search (DFS) is a fundamental graph searching technique known since the 19th century (see for instance Luca's report on Trémaux's work [10]) and popularized by Hopcroft and Tarjan [4, 11 in the seventies. The structure of DFS enables efficient algorithms for many other graph problems [12]. Performing a DFS on a graph defines a spanning tree with specific properties (also known as a Trémaux tree) and an embedding of it as a rooted planar tree, the edges going out of a vertex being circularly ordered according to the discover order of the DFS).

A rooted spanning tree $T$ of a graph $G$ defines a partition of the edge set of $G$ into two classes, the set of tree edges $E(T)$ and the set of cotree-edges $E(G) \backslash E(T)$. It also defines a partial order $\preceq$ on $V(G): x \preceq y$ if the tree path linking $y$ to the root of $T$ includes $x$. The rooted tree $T$ is a Trémaux tree if every cotree edge is incident to two comparable vertices (with respect to $\preceq$ ). A Trémaux tree $T$ defines an orientation of the edges of the graph: an edge $\{x, y\}$ (with $x \prec y$ ) is oriented from $x$ to $y$ (upwards) if it is a tree edge and from $y$ to $x$ (downwards) if it is a cotree edge. Cotree edges of a Trémaux tree are called back edges. We will denote by $\omega^{+}(v)$ the set of the edges incident to a vertex $v$ and going out of $v$. When $T$ is a Trémaux tree, the partial order $\preceq$ is extended to $V(G) \cup E(G)$ (or to $G$ for short) as follows: for any edge $e=(x, y)$ oriented 
from $x$ to $y$, put $x \prec e$ and if $x \prec y$ (that is: if $e$ is a tree-edge) also put $e \prec y$.
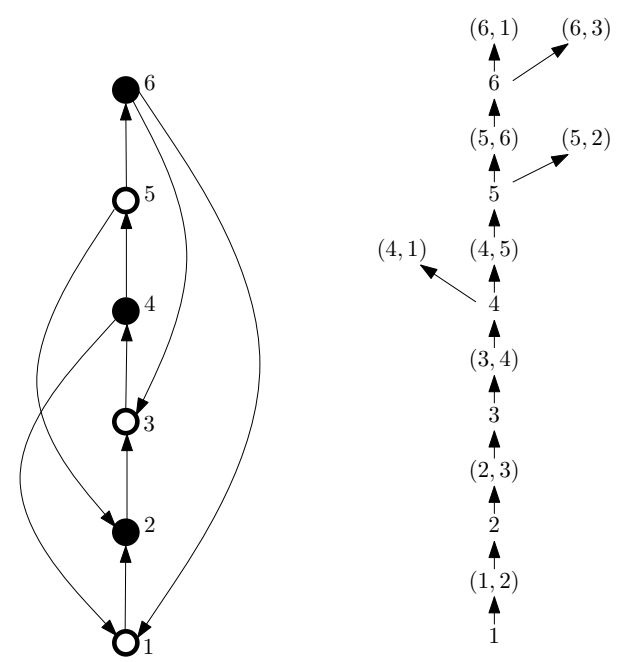

Figure 1: The partial order $\prec$ defined by a Trémaux tree of $K_{3,3}$ (cover relation correspond to bottom-up arrows as usual).

Notice that in the partial order $\prec$, all elements $\alpha$ and $\beta$ of $G$ have an unique greatest lower bound (meet) $\alpha \wedge \beta$. Moreover, the maximal chains of $\prec$ all have the same structure: they begin with the root vertex of $T$, alternate between vertices and edges, and include at most one back-edge (which is then the maximum of the chain).

When $\alpha \preceq \beta$, the unique chain (of $\prec$ ) with minimum $\alpha$ and maximum $\beta$ which is maximal (with respect to set-inclusion) is denoted by $[\alpha ; \beta]$.

It will be helpful to introduce a notation for the minimal element of the interval $] \alpha ; \beta]$ :

Definition 2.1 For $x \prec e$, where $x \in V(G)$ and $e \in E(G)$ we define

$$
\operatorname{stem}(x, e)=\min ] x ; e]
$$

This means that $f$ is the first edge in the unique chain of $\prec$ with minimum $x$ and maximum e.

Notice that in this definition, as in the remaining of the paper, intervals and "min" will always be related to the partial order $\prec$ defined by the considered Trémaux tree.

Definition 2.2 The function low $: G \rightarrow V(G)$ is defined by

$$
\operatorname{low}(\alpha)=\min (\{\alpha\} \cup\{v \in V(G): \exists(u, v) \in E(G) \backslash E(T),(u, v) \succeq \alpha\}) .
$$

Notice that this function is well defined and that $\operatorname{low}(\alpha) \preceq \alpha$. 
In the literature, this function is usually only defined on $V(G)$. Our extension is such that for any edge $e=(x, y)$ :

$$
\text { low }(e)= \begin{cases}\min (x, \text { low }(y)) & \text { if } e \text { is a tree edge } \\ y & \text { if } e \text { is a back-edge }\end{cases}
$$

Definition 2.3 The fringe Fringe $(e)$ of an edge $e=(x, y)$ is defined by:

$$
\text { Fringe }(e)=\{f \in E(G) \backslash E(T): f \succeq e \text { and } \operatorname{low}(f) \prec x\}
$$

Definition 2.4 The low set $\mathrm{L}(\alpha)$ of $\alpha \in V(G) \cup E(G)$ is defined by:

$$
\mathrm{L}(\alpha)=\{f \in E(G) \backslash E(T): f \succeq \alpha \text { and } \operatorname{low}(f)=\operatorname{low}(\alpha)\}
$$

Notice that $\mathrm{L}(e)=\{e\}$ if $e$ is a back-edge.

Definition 2.5 Given a tree edge $e \in E(T)$, we will call $e=(x, y)$ :

- $a$ block edge if $x \preceq \operatorname{low}(y)$, that is if $\operatorname{low}(e)=x$ (this means $e$ is either an isthmus or the minimum edge of a block of the graph);

- $a$ thin edge

if low $(y) \prec x$ and there exists no back-edge $(u, v)$ with $u \succeq y$ and $\operatorname{low}(y) \prec u \prec x$;

- $a$ thick edge

if low $(y) \prec x$ and there exists a back-edge $(u, v)$ with $u \succeq y$ and $\operatorname{low}(y) \prec u \prec x$.

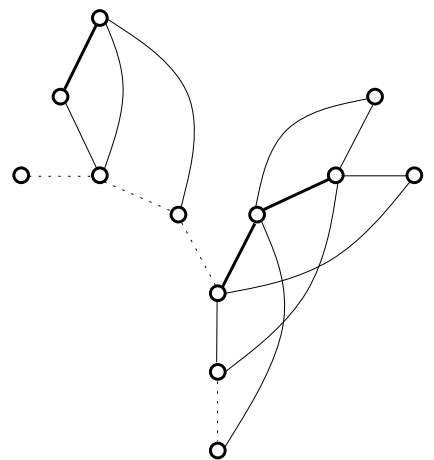

Fig. 2. Block, thin and thick edges. Block edges are dotted, thin edges are light and thick edges are fat.

Definition 2.6 A TT-precedence order $\prec^{\star}$ is a partial order on $E(G)$ such that, for any $v \in V(G)$ and any $e, f \in \omega^{+}(v)$ :

- if $\operatorname{low}(e) \prec \operatorname{low}(f)$ then $e \prec^{\star} f$,

- if low $(e)=\operatorname{low}(f), f$ is a thick tree edge but $e$ is not, then $e \prec^{\star} f$. 


\section{Trémaux Trees and Planarity}

Planarity has been related to Trémaux trees by de Fraysseix and Rosenstiehl in a series of articles [7, 8, 97. One of these characterizations is based on a the existence of a special bipartition of the low angles of the back-edges into left ones and right ones. The constraints that the bipartition has to fulfill is encoded into two relations, namely the $T$-alike and $T$-opposite relations.

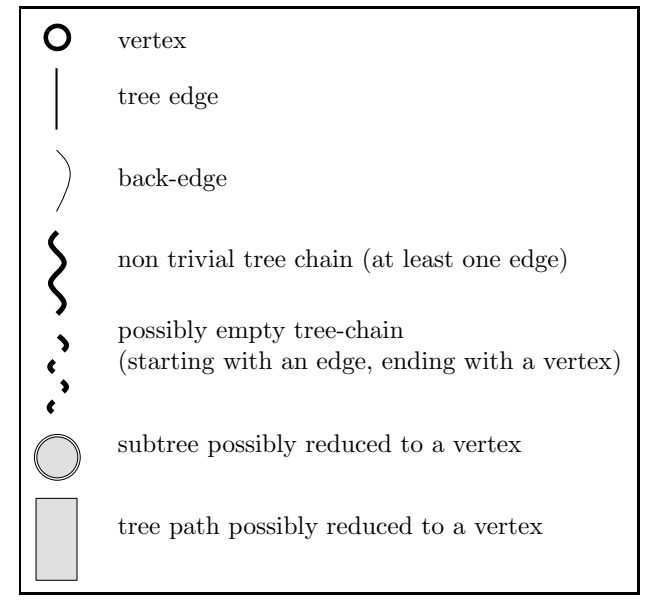

Figure 3: Symbols used in the figures

We don't give here the formal definition of $T$-alike and $T$-opposite relations in terms of $\prec$, but simply recall the characterization given in 7 :

Theorem 1 Let $G$ be a graph with Trémaux tree $T$. Then $G$ is planar if and only if there exists a partition of the back-edges of $G$ into two classes so that any two edges belong to a same class if they are T-alike and any two edges belong to different classes if they are T-opposite.

Instead of working with this characterization, we introduce an equivalent characterization based on a single configuration.

Definition 3.1 Let $v$ be a vertex and let $e_{1}, e_{2} \in \omega^{+}(v)$.

The interlace set $\operatorname{Interlaced}\left(e_{1}, e_{2}\right)$ is defined by:

$$
\operatorname{Interlaced}\left(e_{1}, e_{2}\right)=\left\{f \in \operatorname{Fringe}\left(e_{1}\right): f \succ \operatorname{low}\left(e_{2}\right)\right\}
$$

Definition 3.2 Given a graph $G$ and a Trémaux tree $T$ of $G$, a coloring $\lambda$ : $E(G) \backslash E(T) \rightarrow\{-1,1\}$ is an $\mathrm{F}$-coloring if, for every vertex $v$ and any edges $e_{1}, e_{2} \in \omega^{+}(v), \operatorname{Interlaced}\left(e_{1}, e_{2}\right)$ and $\operatorname{Interlaced}\left(e_{2}, e_{1}\right)$ are monochromatic and colored differently. 


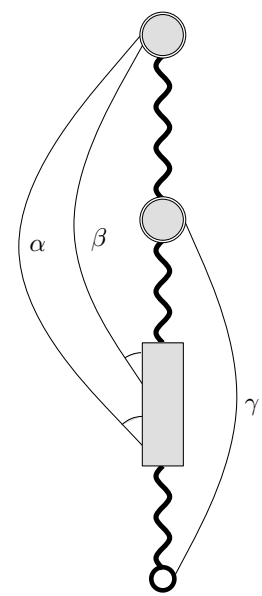

case (i)

$\alpha$ and $\beta$ are $T$-alike

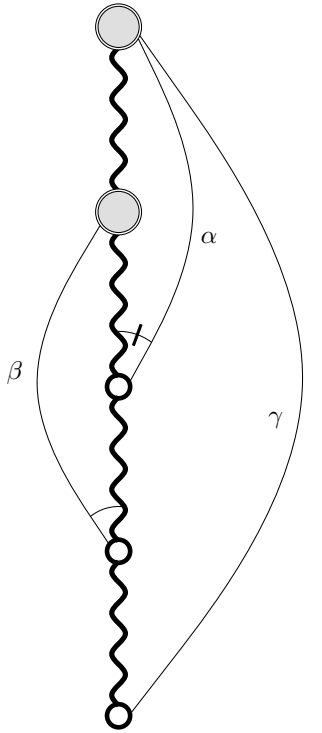

case (ii)

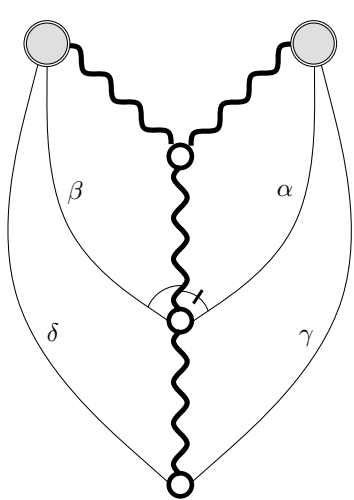

case (iii)

Figure 4: Definition of $T$-alike and $T$-opposite relations

It is easily checked that a coloring is an $F$-coloring if and only if two $T$-alike back-edges are colored the same and two $T$-opposite back-edges are colored differently.

In a planar drawing, an $F$-coloring is defined by the partition of the backedges on two sets, the edges $f$ having their low incidence on the left (resp. the right) of the tree edge stem(low $(f), f)$. The following lemma is straightforward and does not deserve a proof:

Lemma 1 Let $G$ be a planar graph with Trémaux tree $T$. Then $G$ has an $F$ coloring.

Definition 3.3 An F-coloring $\lambda: E(G) \backslash E(T) \rightarrow\{-1,1\}$ is strong if, for any $v \in V(G)$ the low set $\mathrm{L}(v)$ is monochromatic.

Lemma 2 If $G$ has an F-coloring then it has a strong F-coloring.

The addition of the constraints that the sets $\mathrm{L}(v)$ are monochromatic may not lead to a contradiction, as the only constraints involving $f_{1} \in \mathrm{L}(v)$ would also involve any $f_{2} \in \mathrm{L}(v)$ and would require that $f_{1}$ and $f_{2}$ actually have the same color.

¿From the "low angles bicoloration" $\lambda$ we define a "high angles bicoloration" $\widehat{\lambda}$ on the whole edge set of the graph (on both tree edges and back-edges). 
Definition 3.4 Let $\lambda: E(G) \backslash E(T) \rightarrow\{-1,1\}$ be a strong F-coloring. We define the coloring $\widehat{\lambda}: E(G) \rightarrow\{-1,1\}$ by:

$$
\widehat{\lambda}(e)= \begin{cases}\lambda(e), & \text { if } e \text { is a back-edge } \\ \lambda(f), & \text { if } e \text { is a tree edge and } f \in \text { Fringe }(e) \text { with maximal low }(f)\end{cases}
$$

Lemma 3 Let $G$ be a graph, let $T$ be a Trémaux tree of $G$, let $\lambda$ be a strong $F$-coloring and let $\hat{\lambda}$ be the associated mapping. We define the circular order of the edges at a vertex $v$ as follows:

Let $e_{1} \succ^{\star} e_{2} \succ^{\star} \ldots \succ^{\star} e_{p}$ be the edges in $\omega^{+}(v)$ with $\widehat{\lambda}\left(e_{i}\right)=-1$ and let $e_{p+1} \prec^{\star} e_{p+2} \prec^{\star} \ldots \prec^{\star} e_{q}$ be the edges in $\omega^{+}(v)$ with $\widehat{\lambda}\left(e_{i}\right)=1$.

In the circular order around $v$ one finds the incoming tree edge (if $v \neq r$ ) and then $L_{1}, e_{1}, R_{1}, L_{2}, e_{2}, R_{2}, \ldots, L_{q}, e_{q}, R_{q}$ where $L_{i}$ (resp. $R_{i}$ ) is the set of incoming cotree edges $f=(x, v)$ such that $\lambda(f)=-1$ (resp. $\lambda(f)=1)$ and the tree-path linking $r$ to $x$ includes $e_{i}$. For $e_{i}, e_{j} \in L_{k}$ (resp. for $\left.e_{i}, e_{j} \in R_{k}\right)$, one finds $e_{i}$ before (resp. after) $e_{j}$ in the circular order if $\operatorname{stem}\left(e_{i} \wedge, e_{j}, e_{i}\right) \prec^{\star}$ $\operatorname{stem}\left(e_{i} \wedge e_{j}, e_{j}\right)$.

Then these circular orders define a planar embedding of $G$.

In a drawing where the tree edges cross no other edges, only two kind of crossings could occur:
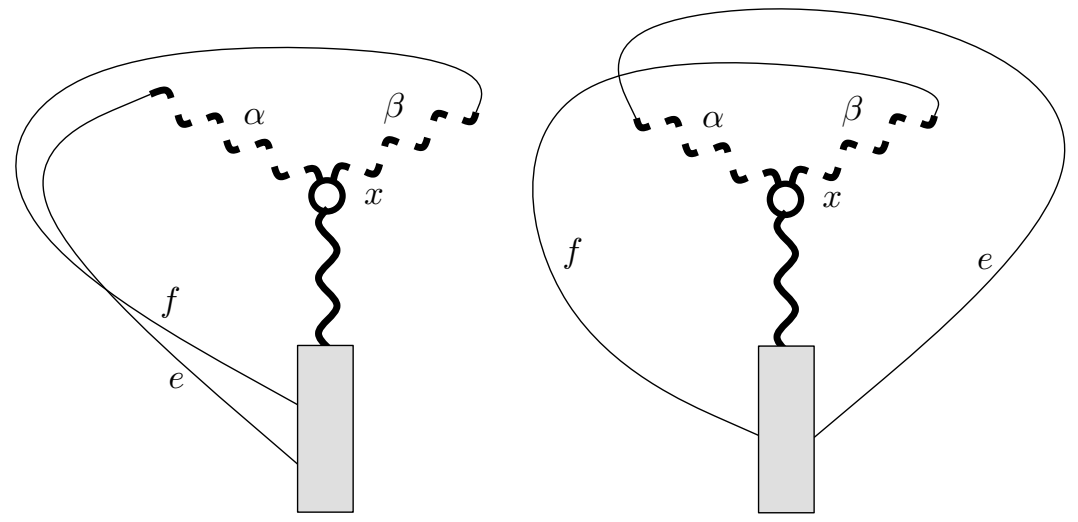

Let $x=e \wedge f, y=\operatorname{low}(f), z=\operatorname{low}(e), \alpha=\operatorname{stem}(x, e)$ and $\beta=\operatorname{stem}(x, f)$. Without loss of generality we assume $\lambda(f)=-1$.

- The first case corresponds to two back-edges $e, f$ with $\lambda(e)=\lambda(f)$ and $\operatorname{low}(e) \leq \operatorname{low}(f)$.

If $\operatorname{low}(\alpha)=\operatorname{low}(\beta)=\operatorname{low}(e)=\operatorname{low}(f)=u$, then the contradiction arises from the definition of the circular order at $u$. Otherwise, as $f \in$ Interlaced $(\beta, \alpha), f$ is colored the same way as the back-edge in Fringe $(\beta)$ with maximal low-value. Hence $\widehat{\lambda}(\beta)=\lambda(f)=-1$. According to the definition of the circular order at $x, \widehat{\lambda}(\alpha))=-1$ and $\alpha \succ^{\star} \beta$. According to the thin-thick precedence of $\prec^{\star}$ it follows that the edge $e^{\prime} \in$ Fringe $(\alpha)$ 
with maximal low-value is such that $\operatorname{low}\left(e^{\prime}\right) \succ \operatorname{low}(\beta)$, that is: $e^{\prime} \in$ Interlaced $(\alpha, \beta)$. Thus $\widehat{\lambda}(\alpha)=\lambda\left(e^{\prime}\right) \neq \lambda(f)$, a contradiction.

- The second case corresponds to two back-edges $e, f$ with $\lambda(e) \neq \lambda(f)$. By symmetry we may assume low $(\alpha) \leq \operatorname{low}(\beta)$ and $\lambda(e)=1$.

Then $\operatorname{low}(\beta) \succ \operatorname{low}(x)$ as $\lambda$ is a strong $F$-coloring. Thus $f$ is colored the same way as the back-edge in Fringe $(\beta)$ with maximal low-value and $\widehat{\lambda}(\beta)=\lambda(f)=-1$. According to the definition of the circular order at $x$, $\widehat{\lambda}(\alpha))=-1$ and $\alpha \succ^{\star} \beta$. It follows that $e \in \mathrm{L}(x)$, a contradiction.

Theorem 2 Let $G$ be a graph, let $T$ be a Trémaux tree of $G$. The following conditions are equivalent:

(i) $G$ is planar,

(ii) $G$ admits an F-coloring,

(iii) $G$ admits a strong F-coloring.

Moreover, if $G$ is planar, any strong $F$-coloring $\lambda$ defines a planar embedding of $G$ in which a back-edge $e$ has its lowest incidence to the left of the tree if $\lambda(e)=-1$ and to the right of the tree if $\lambda(e)=1$

This is a direct consequence of the previous lemmas.

\section{The Planarity Testing Algorithm}

\subsection{Outline}

Let $G$ be a graph of size $m$. The three steps are performed in $O(m)$-time. The first step is composed of a preliminary DFS on $G$ and the computation of the low function and the status of the edges (block/thin/thick). The second step is the computation of a $T T$-precedence order, which may be efficiently performed using a bucket sort. We now examine the last step of the algorithm, which tests the planarity of the graph.

We shall consider some data structure CS responsible for maintaining a set of bicoloration constraints on a set of back-edges. We assign to each edge of the graph $e$ such a data structure $\operatorname{CS}(e)$. These structures are initialized as follows: $\mathrm{CS}(e)$ is empty if $e$ is a tree edge and includes $e$ (with no bicoloration constraints) if $e$ is a back-edge. We say that all the back-edges have been processed and that the tree edges are still unprocessed.

- While there exists a vertex $v$, different from the root, such that all the edges in $\omega^{+}(v)$ have been processed. Let $e=(u, v)$ be the tree edge entering $v$. Let $e_{1} \prec^{\star} e_{2} \prec^{\star} \ldots \prec^{\star} e_{k}$ be the edges in $\omega^{+}(v)(k \geq 1)$. We do the following: 
- Initialize $\operatorname{CS}(e)$ with $\operatorname{CS}\left(e_{1}\right)$.

- For $i: 2 \rightarrow k$, Merge $\operatorname{CS}\left(e_{i}\right)$ into $\operatorname{CS}(e)$, that is: add to $\operatorname{CS}(e)$ the edges in $\operatorname{CS}\left(e_{i}\right)$ and add the $F$-coloring constraints corresponding to the pairs of edges $e_{j}, e_{i}$ with $j<i$ (notice that all the concerned back-edges belong to $\mathrm{CS}(e)$ ). If some constraint may not be satisfied, the graph is declared non-planar.

- Remove from all of the CS(e) every back-edge with lower incidence $u$.

- We declare that edge $e$ has been processed.

- As all the edges have been processed, we declare that the graph is planar.

\subsection{Data-structure, Complexity and Embedding Compu- tation}

First notice that the processing order of the tree edges is simple to compute, using either a "topological sort technique" (by maintaining the unprocessed outdegree of vertices) or by following the backtrack order of a DFS steered by the TT-order. Also, we should notice that the structure $\operatorname{CS}(f)$ is only used (after its computation) when computing $\operatorname{CS}(e)$ where $f$ is the predecessor of $f$ in $\prec$. It follows that we may "destroy" $\operatorname{CS}(f)$ when computing $\operatorname{CS}(e)$ without any risks.

The data structure for $\mathrm{CS}(e)$ is a stack of double top-to-bottom linked stacks. This means that each element of $\operatorname{CS}(e)$ is a pair $\left(\mathrm{S}^{0}, \mathrm{~S}^{1}\right)$ of stacks. Each such pair correspond to a complete bipartite constraint graph: all the edges in $S^{i}(i \in$ $\{0,1\}$ ) have to be colored the same and they have to be colored differently from the back-edges in $\mathrm{S}^{j}$ (for $j \neq i$ ). These will be all the constraints encoded by the $\mathrm{CS}(e)$ (hence no constraint exists between back-edges belonging to different stack pairs).

Some additional constraints will allow to get amortized constant time operations: Let $\left(S_{1}^{0}, S_{1}^{1}\right),\left(S_{2}^{0}, S_{2}^{1}\right), \ldots,\left(S_{k}^{0}, S_{k}^{1}\right)$ be the pairs stacked in some $\operatorname{CS}(e)$. Then:

- The top back-edges of $S_{i}^{0}$ of $S_{i}^{1}$ have both a lower incidence which is strictly smaller than the lower incidences of the bottoms of $S_{i+1}^{0}$ and $S_{i+1}^{1}$.

- the lower incidences of the back-edges belonging to some stack $S_{i}^{\alpha}$ are in non-decreasing order.

Notice that, for any tree-edge $e=(u, v), \operatorname{CS}(e)$ will exactly include the backedges $f=(x, y)$ such that $x \succ v$ and $u \succ y$. Hence the set of edges in $\operatorname{CS}(e)$ is Fringe $(e)$.

Now consider the operations performed by the algorithm on the structures $\mathrm{CS}(e)$ :

- Merge of $\operatorname{CS}\left(e_{i}\right)$ into $\operatorname{CS}(e)$ : the structure $\operatorname{CS}(e)$ then contains the backedges in $X\left(e_{i}\right)=\bigcup_{j<i}$ Fringe $\left(e_{j}\right)$ and their bicoloration constraints. By 
induction, the graph of these constraints is a disjoint union of complete bipartite graphs encoded by the pairs of stacks. The $F$-coloring conditions now express as:

- all the back-edges in $\operatorname{CS}(e)$ (i.e. in $X\left(e_{i}\right)$ ) which have a lower incidence strictly greater than low $\left(e_{i}\right)$ should have the same color,

- all the back-edges in $\operatorname{CS}\left(e_{i}\right)$ which have a lower incidence strictly greater than low $(v)$ should have the same color,

- the two above sets of edges should be colored differently.

These constraints are added as follows:

- let $a$ be the smallest lower incidence of the back-edges in CS(e) (according to monotonicity we just have to look at the bottom backedges of the stacks of the bottom pair). Then $a=\operatorname{low}(v)$.

- let $b$ be the smallest lower incidence of the back-edges in $\operatorname{CS}\left(e_{i}\right)$ (as above, this is computed in constant time). Then $b=\operatorname{low}\left(e_{i}\right)$.

- if $\operatorname{CS}(e)=\left(\left(S_{1}^{0}, S_{1}^{1}\right),\left(S_{2}^{0}, S_{2}^{1}\right), \ldots,\left(S_{k}^{0}, S_{k}^{1}\right)\right)$, let $j$ be the biggest integer $\leq k$ such that none of the back-edges in the top of $S_{j}^{0}$ and $S_{j}^{1}$ have a lower incidence greater than $b$. Then it should be checked that for $j+1<j^{\prime} \leq k$, one of $S_{j^{\prime}}^{0}$ and $S_{j^{\prime}}^{1}$ is empty (for otherwise, we have found a contradiction in the constraints proving that $G$ is not planar). By flipping pairs if necessary we assume $S_{j^{\prime}}^{1}$ is empty. Moreover, only one of $S_{j+1}^{0}$ and $S_{j+1}^{1}$ contains back-edges whose lower incidence is greater than $b$ (for otherwise we have a contradiction proving that $G$ is not planar). Up to a flipping of the pair of stack, we may assume this is $S_{j}^{0}$. Then we fuse $S_{j}^{0}, \ldots, S_{k}^{0}$ into $S_{j}^{0}$ and $\operatorname{CS}(e)=\left(\left(S_{1}^{0}, S_{1}^{1}\right),\left(S_{2}^{0}, S_{2}^{1}\right), \ldots,\left(S_{j}^{0}, S_{j}^{1}\right)\right)$.

- similarly, if $\operatorname{CS}\left(e_{i}\right)=\left(\left(T_{1}^{0}, T_{1}^{1}\right),\left(T_{2}^{0}, T_{2}^{1}\right), \ldots,\left(T_{p}^{0}, T_{p}^{1}\right)\right)$, let $q=2$ if $T_{1}^{0}$ or $T_{1}^{1}$ has a bottom edge whose lower incidence is $a$ and let $q=1$ otherwise. Then it should be checked that for $q \leq q^{\prime} \leq p$, one of $S_{q^{\prime}}^{0}$ and $S_{q^{\prime}}^{1}$ is empty (for otherwise, we have found a contradiction in the constraints proving that $G$ is not planar). By flipping pairs if necessary we assume $S_{q^{\prime}}^{0}$ is empty. It should be noticed that if $q=2$, then one of $T_{1}^{0}$ and $T_{1}^{1}$ is empty. By flipping the pair if necessary, we may assume this is $T_{1}^{0}$. Then $T_{1}^{1}$ contains exactly the back-edges in $\mathrm{L}\left(e_{i}\right)$.

Then we fuse $T_{q}^{1}, \ldots, T_{p}^{1}$ into $S_{j}^{1}$ and, if $q=2$, we add to the one of $S_{1}^{0}$ and $S_{1}^{1}$ which is not empty the edges from $T_{1}^{0}$.

As the number of pairs of stacks decreases at each "fuse", as no new pair of stacks is created and as the initial number of pairs of stacks is $O(m)$, the global time spent in this step by the algorithm is $O(m)$.

- Deletion of the back-edges whose lower incidence is $u$ : by monotonicity, we only have to check the top of the stacks in the top pair of stacks. As 
every back-edge will be deleted exactly once by the algorithm, the global time spent for deletions is $O(m)$.

As the stacks used by the algorithm have their elements linked in a topbottom manner, it is easy to keep, while "deleting" back-edges, the link between edges that have to get the same $\lambda$-value and to add a special type of links between some edges which have to get different $\lambda$-value. This way, a spanning forest of the constraint graph is maintained with a constant time cost per deletion, which allows to propagate $\lambda$-values after the planarity testing phase. A $\lambda$-value for each back-edge being computed, the embedding follows and is easily computed in linear time. 


\subsection{Example}

We consider a sample planar graph with an arbitrary Trémaux tree (see Fig. 5). The right of the figure display the information computed by the two first steps of the algorithm: the status of the edges (block/thin/thick) and a TT-precedence order $\prec^{\star}$, represented here as a circular order of the outgoing edges.
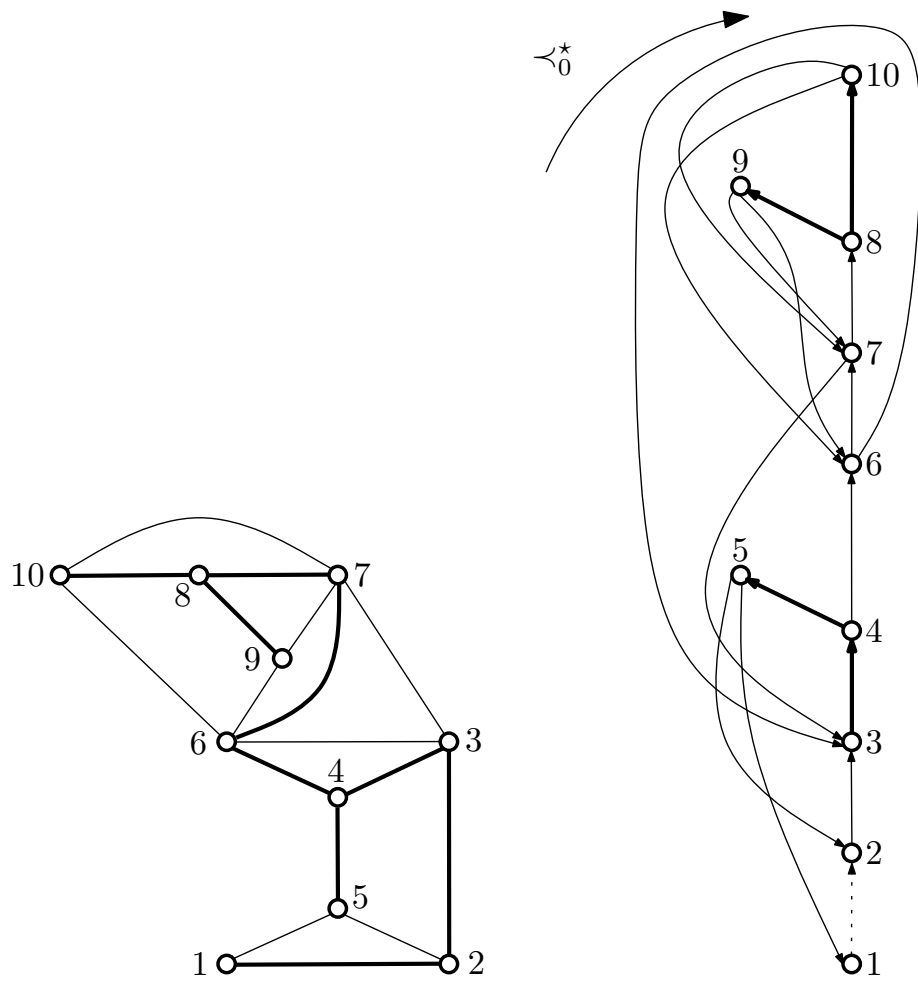

Figure 5: A sample graph with a Trémaux tree $T$ (on the left). The block/thin/thick partition and a $T T$-precedence order $\prec^{\star}$ (on the right).

The algorithm first computes the CS's of the back-edges. Then it proceeds by iterating on the vertices whose outgoing edges are all processed. At each such vertex $v$, the CS of the incoming tree edge is computed, using a Merge and possibly a Deletion step (denoted by $\rightarrow$ ). In the following table, the CS's are represented as sequences of double stacks. 


$$
\begin{aligned}
& v=9 \quad: \operatorname{CS}((8,9)) \quad=\lfloor(9,6)\rfloor \quad,\lfloor(9,7)\rfloor \\
& v=10 \quad: \quad \operatorname{CS}((8,10))=\lfloor(10,6)\rfloor \quad\lfloor,\lfloor(10,7)\rfloor \\
& v=5 \quad: \operatorname{CS}((4,5))=\lfloor(5,1)\rfloor \quad,\lfloor(5,2)\rfloor \\
& \left.v=8 \quad: \quad \operatorname{CS}((7,8))=\begin{array}{c}
(10,6) \\
(9,6)
\end{array}\right\rfloor \quad\lfloor,\lfloor(9,7)\rfloor(10,7) \\
& \rightarrow \begin{array}{c|c|c}
(10,6) \\
(9,6)
\end{array} \mid \quad \text { (Deletion) } \\
& v=7 \quad: \quad \operatorname{CS}((6,7))=\lfloor(7,3)\rfloor \quad, \quad \begin{array}{c}
(10,6) \\
(9,6)
\end{array} \\
& \rightarrow\lfloor(7,3) \perp \quad \text { (Deletion) } \\
& v=6 \quad: \operatorname{CS}((4,6))=\left|\begin{array}{l}
(6,3) \\
(7,3)
\end{array}\right| \\
& v=4 \quad: \operatorname{CS}((3,4))=\lfloor(5,1)\rfloor,\lfloor(5,2)\rfloor \quad, \quad\left\lfloor\begin{array}{l}
(6,3) \\
(7,3)
\end{array} \mid\right. \\
& \rightarrow\lfloor(5,1) \perp \quad, \quad\lfloor(5,2) \perp \quad \text { (Deletion) } \\
& v=3 \quad: \operatorname{CS}((2,3))=\lfloor(5,1)\rfloor \quad,\lfloor(5,2)\rfloor \\
& \rightarrow\left\lfloor(5,1) \_\right. \text {(Deletion) } \\
& v=2 \quad: \operatorname{CS}((1,2))=\lfloor(5,1)\rfloor \\
& \rightarrow \emptyset \quad \text { (Deletion) }
\end{aligned}
$$

\section{References}

\section{References}

[1] H. de Fraysseix and P. Ossona de Mendez. PIGALE: Public Implementation of a Graph Algorithm Library and Editor. Free Software (GPL licence), 2002. http://pigale. sourceforge.net.

[2] H. de Fraysseix and P. Ossona de Mendez. PIGALE. In R. Tamassia, editor, Handbook of Graph Drawing and Visualization, chapter 26. CRC Press, 2006. in preparation. 
[3] J.M. Boyer, P.F. Cortese, M. Patrignani, and G. Di Battista. Stop minding your P's and Q's: implementing fast and simple DFS-based planarity and embedding algorithm. In Graph Drawing, volume 2912 of Lecture Notes in Computer Science, pages 25-36. Springer, 2004.

[4] J.E. Hopcroft and R.E. Tarjan. Efficient algorithms for graph manipulation. Communications of the ACM, (16):372-378, 1973.

[5] J.E Hopcroft and R.E. Tarjan. Efficient planarity testing. J. Assoc. Comput. Math., 21:549-568, 1974.

[6] S.G. Williamson. Embedding graphs in the plane - algorithmic aspects. Annals of Discrete Mathematics, (6):349-384, 1980.

[7] H. de Fraysseix and P. Rosenstiehl. A characterization of planar graphs by Trémaux orders. Combinatorica, 5(2):127-135, 1985.

[8] H. de Fraysseix and P. Rosenstiehl. Système de référence de Trémaux d'une représentation plane d'un graphe planaire. Annals of Discrete Mathematics, 17:293-302, 1983.

[9] H. de Fraysseix and P. Rosenstiehl. A depth-first search characterization of planarity. Annals of Discrete Mathematics, 13:75-80, 1982.

[10] E. Lucas. Récréations Mathématiques. Paris, 1882.

[11] R.E. Tarjan. Depth-first-search and linear graph algorithm. SIAM J. Comp., 2:146-160, 1972.

[12] S. Even. Graph Algorithms. Computer Science Press, Rockville, MD, 1979. 